\title{
Cloning, characterization of TaGS3 and identification of allelic variation associated with kernel traits in wheat (Triticum aestivum L.)
}

Jian Yang ${ }^{1}$, Yanjie Zhou', Yu'e Zhang ${ }^{1}$, Weiguo Hu' ${ }^{1}$, Qiuhong Wư ${ }^{2}$, Yongxing Chen ${ }^{2}$, Xicheng Wang ${ }^{1}$, Guanghao Guo ${ }^{2}$ Zhiyong Liu' ${ }^{2 *}$ Tingjie Cao ${ }^{1 *}$ (i) and Hong Zhao ${ }^{1}$

\begin{abstract}
Background: Grain weight is an important yield component. Selection of advanced lines with heavy grains show high grain sink potentials and strong sink activity, which is an increasingly important objective in wheat breeding programs. Rice OsGS3 has been identified as a major quantitative trait locus for both grain weight and grain size. However, allelic variation of GS3 has not been characterized previously in hexaploid wheat.

Results: We cloned 2445, 2393, and 2409 bp sequences of the homologs TaGS3-4A, TaGS3-7A, and TaGS3-7D in wheat 'Changzhi 6406', a cultivar that shows high grain weight. The TaGS3 genes each contained five exons and four introns, and encoded a deduced protein of 170, 169, and 169 amino acids, respectively. Phylogenetic analysis of plant GS3 protein sequences revealed GS3 to be a monocotyledon-specific gene and the GS3 proteins were resolved into three classes. The length of the atypical $\mathrm{G} Y$ domain and the cysteine-rich region was conserved within each class and not conserved between classes. A single-nucleotide polymorphism in the fifth exon (at position 1907) of TaGS3-7A leads to an amino acid change (ALA/THR) and showed different frequencies in two pools of Chinese wheat accessions representing extremes in grain weight. Association analysis indicated that the TaGS3-7A-A allele was associated with higher grain weight in the natural population. The TaGS3-7A-A allele was favoured in global modern wheat cultivars but the allelic frequency varied among different wheat-production regions of China, which indicated that this allele is of potential utility to improve wheat grain weight in certain wheat-production areas of China.
\end{abstract}

Conclusions: The novel molecular information on wheat GS3 homologs and the KASP functional marker designed in this study may be useful in marker-assisted breeding for genetic improvement of wheat.

Keywords: TaGS3, Kernel traits, Phylogenetic tree, Functional marker

\section{Background}

Wheat (Triticum aestivum, AABBDD) provides more than $20 \%$ of the calories and $25 \%$ of the protein in the human diet (FAOSTAT; http://Faostat.fao.org/site/339/

\footnotetext{
*Correspondence: zyliu@genetics.ac.cn; caotingjie893@163.com

${ }^{2}$ State Key Laboratory of Plant Cell and Chromosome Engineering, Institute of Genetics and Developmental Biology, Chinese Academy of Sciences, Beijing 100101, China

${ }^{1}$ National Laboratory of Wheat Engineering, Key Laboratory of Wheat Biology and Genetic Breeding in Central Huang-Huai Region, Ministry of Agriculture, Institute of Wheat, Henan Academy of Agricultural Sciences, Zhengzhou 450002, Henan, China
}

default.aspx and http://www.fao.org/worldfoodsituation/ wfs-home/csdben). With the ongoing increase in population size, climate change, and reduction in the available land area, annual gains in crop yields of $\sim 2 \%$ are required to meet food security $[1,2]$. The grain yield of wheat and other cereals is a polygenic trait that is influenced by environmental and genetic interactions at all stages of plant growth [3].

In rice, genes controlling grain weight have been extensively studied [4, 5]. Grain Size 3 (GS3), which encodes a transmembrane protein containing an atypical 
Gy domain and a cysteine-rich region, was the firstcharacterized quantitative trait locus (QTL) that negatively regulates grain length $[6,7] \mathrm{A} \mathrm{C}-\mathrm{A}$ mutation in the second exon of GS3 (A allele) is associated with enhanced grain length in Oryza sativa but is absent in other Oryza species [7]. Pyramiding GL3.3 (Grain Length 3.3) and GW7 (Grain Width 7) enhances yield in an indica hybrid rice background and simultaneously improves yield and grain quality [8]. Grain Size 5 (GS5) encodes a putative serine carboxypeptidase and functions as a positive regulator of grain size and yield [9] Analysis of natural variation indicates that mutation of GS5 is associated with enhanced grain length [10]. Grain width 5 (GW5) encodes a novel nuclear protein of 144 amino acids that is localized to the nucleus and affects grain width [11]. A 1212-bp deletion in rice 'Nipponbare' was selected during japonica domestication, which functions most likely through influencing the expression levels of OsGW5. The GW5 protein is localized to the plasma membrane and can physically interact with and repress the kinase activity of rice GSK2, resulting in accumulation of unphosphorylated OsBZR1 and DLT [12]. A recently reported gene, GLW7 (GRAIN LENGTH AND WEIGHT ON CHROMOSOME 7), also showed a differential expression level in regulating grain weight by allelic variation in the $5^{\prime}$ untranslated region [13]. In addition, Grain Width 6 (GW6) encodes a GNAT-like protein that harbors intrinsic histone acetyltransferase activity, and elevated expression of this gene enhances grain length and weight by enlarging spikelet hulls and accelerating grain filling [14]. Grain Width 2 (GW2) encodes a previously unknown RING-type protein with E3 ubiquitin ligase, which negatively regulates cell division by degrading its substrate(s) by means of the ubiquitinproteasome pathway [15]. DA2 (orthologous to GW2) is an E3 ubiquitin ligase that physically interacts with DA1 in vitro and in vivo in Arabidopsis [16]. Grain Length 3 (GL3) encodes a protein phosphatase with Kelch-like domains (PPKL) family Ser/Thr phosphatase, which shows epistatic interaction with GS3 and OsGSK3 to modulate brassinosteroid signaling to produce extra-long grains in rice [17-19]. The Kelch domains are essential for OsPPKL1 (GL3) biological function [20]. DENSE AND ERECT PANICLE 1 (DEP1) encodes a highly cysteinerich $\mathrm{G}$ protein gamma subunit protein. Gain-of-function mutation of DEP1 enhances meristematic activity, resulting in reduction in the length of the inflorescence internodes, an increased number of grains per panicle, and a consequent increase in grain yield [21].

In wheat, the large genome size and complex genome composition renders map-based cloning of functional genes difficult, especially for complex traits. However, the close relationship between wheat and rice provides the opportunity for researchers to clone homologous functional genes with a common ancestor, in particular for developmentrelated traits [22-24]. On the basis of this theory, a number of grain weight-related genes have been cloned and characterized in natural populations and/or bi-parental populations. Orthologs of TaGW2 have been widely characterized in wheat. Two single-nucleotide polymorphisms (SNPs) forming a Hap-6A-A haplotype for TaGW2-6A are associated with broader grains and higher 1000-grain weight [25]. An additional haplotype, Hap-6B-1 for TaGW2-6B, shows stronger effects on grain weight than TaGW2-6A and this was further validated by gene-editing mutant analysis [26, 27]. A number of reverse genetic studies have shown that $T a G W 2$ has a negative influence on grain size in hexaploid and tetraploid wheat $[28,29]$. Homologs of GW5 also exert many effects on grain weight and grain size; the haplotype TaGS5-3A-T, based on the SNP T/G in the sixth exon, is significantly correlated with larger grain size, higher 1000kernel weight, and lower plant height, spike length, and internode length below the spike, which is under strong positive selection in Chinese modern wheat breeding [2, 30]. TaGW6, an ortholog of rice $G W 6$, encodes a novel indole-3-acetic acid-glucose hydrolase. Variation among wheat homologs of TGW6 on chromosome arms 3AL and 4AL individually explain $\sim 17 \%$ of TGW variation, and the low-expression alleles are associated with low auxin content and high TGW [31, 32]. TaGW7 is a homolog of a gene encoding a TONNEAU1-recruiting motif protein, which is mainly expressed in young spike tissues, was shown to negatively regulate grain weight and grain width using CRISPR-Cas9 gene editing analysis. This gene regulates seed size probably effects genes through cell division pathways [33, 34]. Notably, a number of grain trait-related genes (e.g., TaGW2, TaGL3, and TaGW8) were mapped in the QTL region that is likely to harbpr the causative genes [35-37].

TaGS3-1D has been cloned in hexaploid wheat and a 40-bp indel predicted to generate a new isoform that is associated with grain weight [38]. However, the hexaploid wheat genome consists of three subgenomes, of which the $\mathrm{D}$ genome generally shows the lowest genetic diversity $[39,40]$. Therefore, we were interested in isolating the two homologous TaGS3 genes in the A and B subgenomes and determining the functional variation for potential use in wheat improvement. For this purpose, we cloned the TaGS3 genes, investigated the evolution of GS3 genes in plants, designed genetic markers for TaGS3, validated its effects in a natural population, and evaluated its distribution in major wheat-production areas of China.

\section{Results}

Isolation of TaGS3 in wheat

In wheat, three copies of TaGS3 on chromosome arms 4AL (TraesCS4A02G474000), 7AS (TraesCS7A02G017700), 
and 7DS (TraesCS7D02G015000) were searched in the Chinese Spring RefSeq v1.0 genome database. Primers were designed based on specific regions for each sequence (Table 1). The primers amplified PCR products 2445,2393 , and $2409 \mathrm{bp}$ in length for TaGS3-4A (GenBank accession: KY888174), TaGS3-7A (GenBank accession: KY888186), and TaGS3-7D (GenBank accession: KY888197) in wheat accession 'Changzhi 6406' (a cultivar that exhibits high grain weight). The lengths of the predicted coding sequences of the three genes were 513,510 , and $510 \mathrm{bp}$, encoding putative proteins of 170,169 , and 169 amino acids, respectively. TaGS3-4A, TaGS3-7A, and TaGS3-7D showed similarity in exon-intron structure to that of OsGS3, which consisted of five exons and four introns (Fig. 1). The degree of similarity between the coding region of OsGS3 and that of TaGS3-4A, TaGS3-7A, and TaGS3-7D was 45.92, 43.94, and $44.87 \%$, respectively. In parallel, the similarity of the deduced amino acid sequences was $45.26,44.83$, and $42.24 \%$, respectively.

\section{Phylogenetic analysis of GS3 in plants}

A phylogenetic tree was reconstructed for 14 deduced amino sequences for GS3 homologs from Aegilops tauschii, Hordeum vulgare, Panicum hallii, Setaria italica, Triticum aestivum, Zea mays, Triticum urartu, Sorghum bicolor, Glycine max, and Arabidopsis thaliana. A reported atypical $\mathrm{G} \gamma$ domain gene, $D E P 1$, was used as the outgroup. In the phylogenetic tree the sequences were resolved into two main groups, which comprised the GS3 orthologs and DEP1 orthologs (Fig. 2a). The GS3 orthologous group consisted of ten sequences that were all derived from monocotyledons, whereas the DEP1-orthologous group comprised sequences derived from monocotyledons and dicotyledons.

In the GS3 orthologs group, the relationships among the genes were congruent with the species phylogeny, except that the relationship between TaGS3-7A in wheat subgenome $A$ and TuGS-7A in Triticum urartu was more distant than that with GS3 of Hordeum vulgare. The similarity of the conserved domain among the sequences was $85.14 \%$ overall and the sequences were of similar length (60-66 amino acids) except for that of $T$. urartu (46), whereas the length of the cysteine-rich region was variable and was divided into three classes. Class I consisted of Triticeae species with a conserved length of 92-94 amino acids (85 in Triticum urartu was an exception); Class II included Oryza species of which the longest sequence was 150 amino acids; and Class III consisted of Panicoideae species with a conserved length of 111-116 amino acids. Comparison of the atypical Gy subunit conserved domain among the GS3 ortholog sequences revealed that all sequences contained an identical amino acids fragment "PRP--RLQLAVDALHR-FLEGEI" ("--" represents a non-conserved region and the number of "-" does not reflect the number of amino acids).

\section{Polymorphism of TaGS3-4A and TaGS3-7A among wheat accessions}

Sequences for TaGS3-4A and TaGS3-7A from ten wheat accessions were obtained. To avoid possible false variation generated by sequencing error, an advanced analysis for detection of real variants resulted in a minor allele frequency $>0.1$ (real SNPs must be detected at least twice in ten sequences). In total 17 and 18 variations for TaGS3-4A and TaGS3-7A was detected by multiple alignment, respectively. There were $3,1,6,0,3$, $0,0,0,0,2$, and 2 variations located in the $5^{\prime}$ upstream region, first exon, first intron, second exon, second intron, third exon, third intron, fourth exon, fourth intron, fifth exon, and the 3' downstream region for TaGS3-4A, respectively. In parallel, $4,0,2,0,4,0,0,1,3,3$, and 1 variable sites were detected in the corresponding regions for TaGS3-7A (Tables 2 and 3). Among them, both three SNPs causing changes causing amino acid change were determined for each of TaGS3-4A and TaGS3-7A, which were located in the first exon (1) and fifth exon (2), and in the fourth exon (1) and fifth exon (2), respectively. The variant (GC/AT) at position 70 for TaGS3-4A was located in an atypical Gy subunit, of which the GC allele

Table 1 Primers used in this study

\begin{tabular}{llll}
\hline Primer & Sequence (5'-3') & explanation & predicted PCR product Size \\
\hline TaGS3-4A-F & CGATCCTTCTCTGCGGCAAG & primers for amplifying TaGS3-4A & 2435 bp \\
TaGS3-4A-R & CCTACAGACCGACGACTTCCTG & & 2389 bp \\
TaGS3-7A-F & CGACTTCCTGTCTCCTTCCGG & primers for amplifying TaGS3-7A & 2409 bp \\
TaGS3-7A-R & TCATGCCCGTCAAAAACACCAG & & \\
TaGS3-7D-F & GACGACTTCCTGTCTCCTACTTCC & primers for amplifying TaGS3-7D & \\
TaGS3-7D-R & TCATGCCCGTCAAAAACACCAG & & \\
TaGS3-7A-1907-F1 & FAM-GCGCCGGTTGCTCCTCATCTTGCG & KASP marker for distinguishing A/G allele \\
TaGS3-7A-1907-F2 & HEX-GCGCCGGTTGCTCCTCATCTTGCA & & \\
TaGS3-7A-1907-CR & AGGGACGCCRCCGCAGCACACGGT & & \\
\hline
\end{tabular}




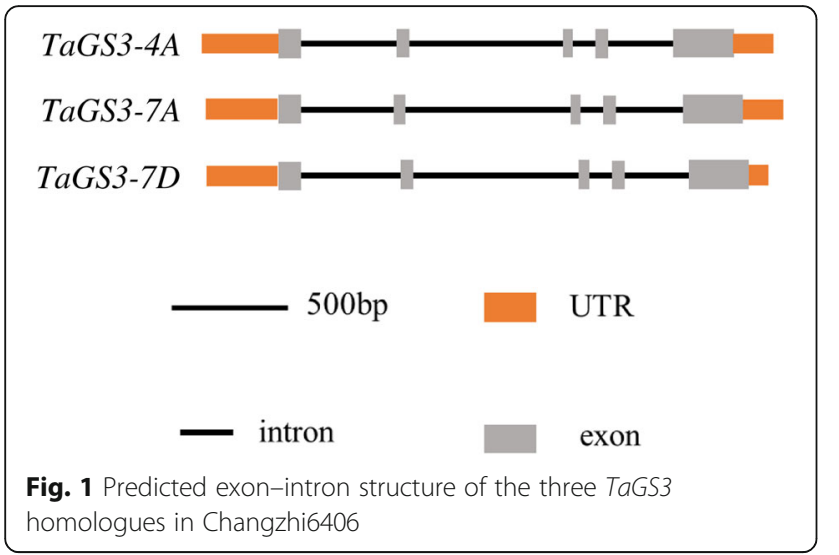

was harbored by 80 and $60 \%$ of accessions grouped into heavy and light grain weight pools, respectively, which indicated this locus was not associated with grain weight. In addition, the frequency of allele A at position 1907 (amino change: ALA/THR) was 0.6 in the heavy grain weight accessions compared with zero in the light grain weight accessions. Therefore, this allele was considered more likely to be associated with grain weight in wheat.

\section{Molecular marker design and validation}

Based on the results of SNP mining, a Kompetitive allele-specific PCR (KASP) marker was designed for the SNP at position 1907 (A/G). The calls for the TaGS3$7 A-A$ allele of the potential light grain weight group were clustered along with the $x$-axis, whereas the calls of the TaGS3-7A-G allele of the potential heavy grain weight group were clustered along with the $y$-axis (Fig. 3 ). Thus, the clustering results clearly distinguished the two alleles, which was used to validate its effects on grain weight in the natural population.

The association between genotypes and grain traits was analyzed based on a mini-core collection (MCC) of 224 wheat accessions for this marker (Additional file 1: Table S1). On the basis of the SNP calls using the KASP marker, significant differences for kernel width, kernel weight, and kernel thickness was observed for the TaGS3-7A-G and TaGS3-7A-A alleles, whereas no significant difference for kernel length was observed (Table 4). In addition, the allele TaGS3-7A-A with superior traits with a rare frequency of $13.107 \%$, which was significantly less than that for TaGS3-7A-G. However, the frequency of TaGS3-7A-A in cultivars (18.085\%) was significantly higher than that in landraces $(0.980 \%)$.

\section{Distribution of the TaGS3-7A-A allele in the major wheat- production areas of China}

In total, 238 wheat cultivars grown in the main wheatproduction areas of China were analyzed to determine the frequency of the TaGS3-7A-A allele (Additional file 1: Table S2). The TaGS3-7A-A allele was detected in $58.4 \%$ of all tested cultivars. Significant differences in the frequency of TaGS3-7A-A were observed among the five province areas. The TaGS3-7A-A allelic frequency

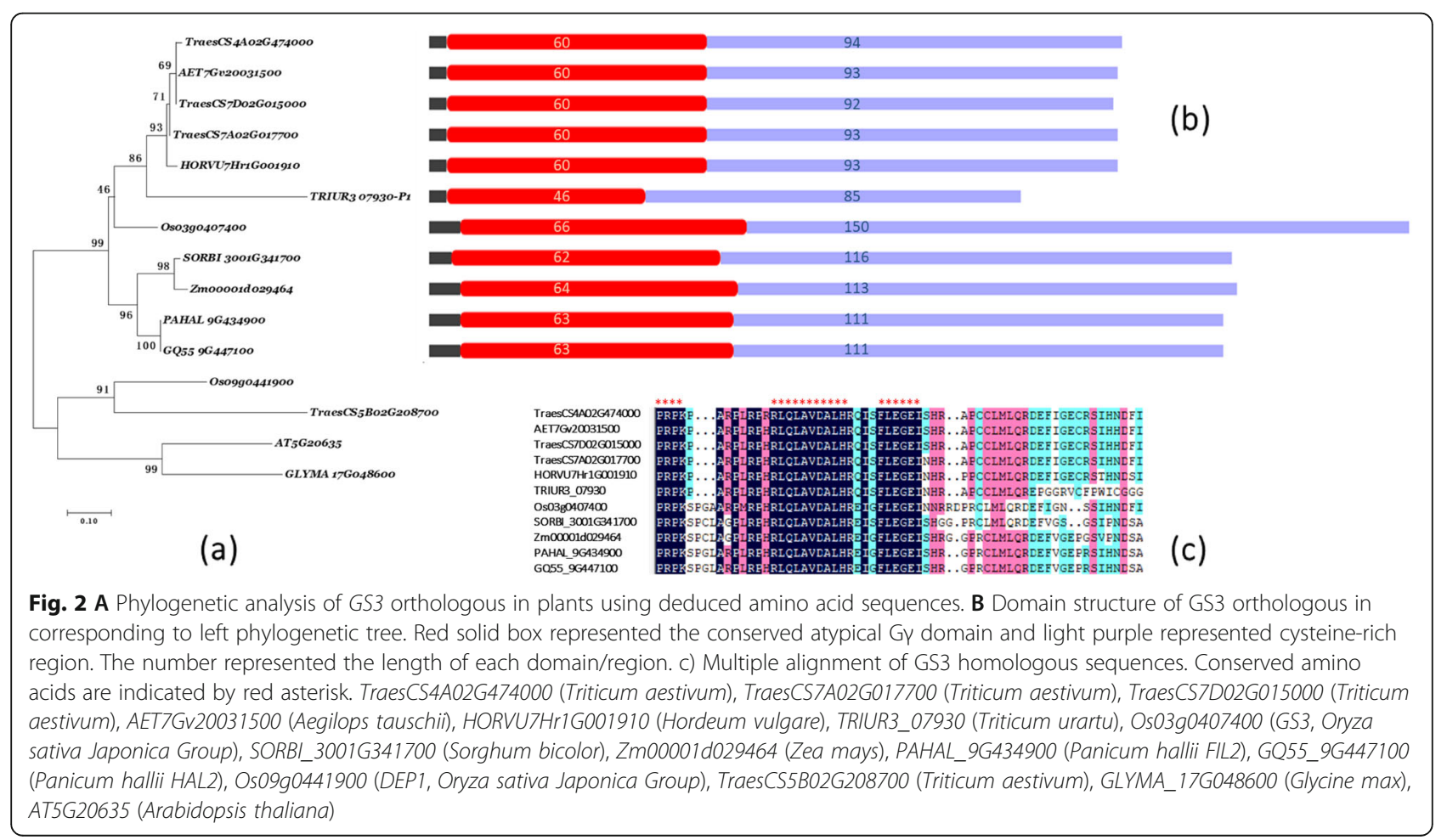


Table 2 Allelic variation of TaGS3-4A. The position was accounted from ATG (1)

\begin{tabular}{|c|c|c|c|c|c|c|c|c|c|c|c|c|}
\hline position & location & $\begin{array}{l}\text { Changzhi } \\
6406\end{array}$ & Sankecun & $\begin{array}{l}\text { Shannong } \\
7859\end{array}$ & Kangdingxiaomai & $\begin{array}{l}\text { Xingyi } \\
4\end{array}$ & Youmangbaifu & $\begin{array}{l}\text { Dongnong } \\
101\end{array}$ & $\begin{array}{l}\text { Gansu } \\
96\end{array}$ & $\begin{array}{l}\text { Fuzhuang } \\
30\end{array}$ & $\begin{array}{l}\text { Bima } \\
4\end{array}$ & $\begin{array}{l}\text { amino } \\
\text { acid } \\
\text { change }\end{array}$ \\
\hline-306 & $5^{\prime} U T R$ & $T$ & $C$ & C & $\bar{T}$ & $T$ & $T$ & $C$ & $T$ & $T$ & $\mathrm{~T}$ & - \\
\hline-129 & $5^{\prime} U T R$ & $\mathrm{TG}$ & CG & TG & TG & TG & TG & CA & CG & TG & TG & - \\
\hline-53 & $5^{\prime} U T R$ & G & A & G & G & G & G & A & A & G & G & - \\
\hline 70 & $\begin{array}{l}\text { 1st } \\
\text { exon }\end{array}$ & GC & AT & GC & GC & GC & GC & AT & AT & GC & GC & $\mathrm{A} / \mathrm{M}$ \\
\hline 200 & $\begin{array}{l}\text { 1st } \\
\text { intron }\end{array}$ & G & G & G & C & G & G & G & G & C & C & - \\
\hline 250 & $\begin{array}{l}1 \text { st } \\
\text { intron }\end{array}$ & T & T & $\mathrm{T}$ & - & $\mathrm{T}$ & $\mathrm{T}$ & $\mathrm{T}$ & T & - & - & - \\
\hline 331 & $\begin{array}{l}1 \text { st } \\
\text { intron }\end{array}$ & $\mathrm{T}$ & A & $\mathrm{T}$ & $\mathrm{T}$ & $\mathrm{T}$ & $\mathrm{T}$ & A & A & $\mathrm{T}$ & $\mathrm{T}$ & - \\
\hline 354 & $\begin{array}{l}1 \text { st } \\
\text { intron }\end{array}$ & $\mathrm{ACT}$ & G & ACTG & ACTG & G & ACTACTG & G & G & G & G & - \\
\hline 378 & $\begin{array}{l}\text { 1st } \\
\text { intron }\end{array}$ & A & G & A & A & A & A & G & G & A & A & - \\
\hline 397 & $\begin{array}{l}1 \text { st } \\
\text { intron }\end{array}$ & T & $\mathrm{T}$ & $\mathrm{T}$ & $\mathrm{T}$ & C & $\mathrm{T}$ & C & $T$ & $\mathrm{~T}$ & $\mathrm{~T}$ & - \\
\hline 633 & $\begin{array}{l}\text { 2nd } \\
\text { intron }\end{array}$ & C & T & $\mathrm{T}$ & $\mathrm{T}$ & C & C & $\mathrm{T}$ & $T$ & $\mathrm{~T}$ & $\mathrm{~T}$ & - \\
\hline 712 & $\begin{array}{l}\text { 2nd } \\
\text { intron }\end{array}$ & A & $\mathrm{T}$ & $\mathrm{T}$ & A & A & A & $\mathrm{T}$ & $\mathrm{T}$ & A & A & - \\
\hline 1140 & $\begin{array}{l}\text { 2nd } \\
\text { intron }\end{array}$ & $\mathrm{T}$ & $\mathrm{T}$ & $T$ & T & C & $\mathrm{T}$ & $\mathrm{T}$ & C & $\mathrm{T}$ & $\mathrm{T}$ & - \\
\hline 1747 & $\begin{array}{l}5 \text { th } \\
\text { exon }\end{array}$ & $\mathrm{T}$ & C & $\mathrm{T}$ & C & T & $T$ & $\mathrm{~T}$ & $T$ & $\mathrm{~T}$ & $\mathrm{~T}$ & $\mathrm{~L} / \mathrm{P}$ \\
\hline 1897 & $\begin{array}{l}\text { 5th } \\
\text { exon }\end{array}$ & C & C & T & C & T & $\mathrm{T}$ & C & C & C & $C$ & AN \\
\hline 1976 & 3'UTR & C & C & G & C & G & G & C & C & $C$ & C & - \\
\hline 2004 & $3^{\prime} U T R$ & C & G & C & C & C & C & G & G & C & C & - \\
\hline
\end{tabular}

exceeded $50 \%$ in all provinces except Henan (i.e., 92.0\% in Sichuan, 62.5\% in Shandong, 60\% in Hebei, and 52.6\% in Shaanxi). The TaGS3-7A-A allele was detected in 50\% of the cultivars in Shaanxi province. However, a significantly lower frequency of TaGS3-7A-A was detected among cultivars from Henan (28.23\%) compared with the other provinces.

\section{Discussion}

\section{Structure and evolution of GS3}

A number of genes that encode $G$ proteins in rice have been cloned and show a variety of functions in the regulation of organ development $[10,21]$. GS3, which encodes heterotrimeric $\mathrm{G}$ proteins that contain an atypical Gy domain, is a major QTL for grain size $[6,10]$. However, the evolutionary history of GS3 in plants has not been examined previously. In the present study, we performed a phylogenetic analysis of protein sequences using the rice GS3 sequence as a query. There was no GS3 homologs in dicotyledon plant, indicated that GS3 homologs was a monocotyledon-specific gene family. We also determined that the wheat genome contains three GS3 orthologous genes on chromosome arms 7AS, 7DS, and 4AL based on the released 'Chinese Spring' reference genome sequence [22]. The molecular evolution of GS3 was consistent with their species evolution relationship. However, the phylogenetic relationship between TaGS3-7A and TuGS3-7A was more distant than that between TaGS3-7A and other Triticeae species, which probably because TuGS3-7A was located on 4AL/ 7BS translocation that is occurred at polyploidizing progress during diploid wheat to polyploid wheat [41-44].

All GS3 orthologous genes showed a conserved atypical Gy domain with high similarity, whereas the length of the cysteine-rich region was variable. In particular, the length of the cysteine-rich region in Triticeae species was $\sim 60 \%$ and $\sim 80 \%$ the length of that in rice and Panicoideae species, respectively. Although the conserved domain usually determines the function of genes universally, the length of the C-terminal tail may affect 


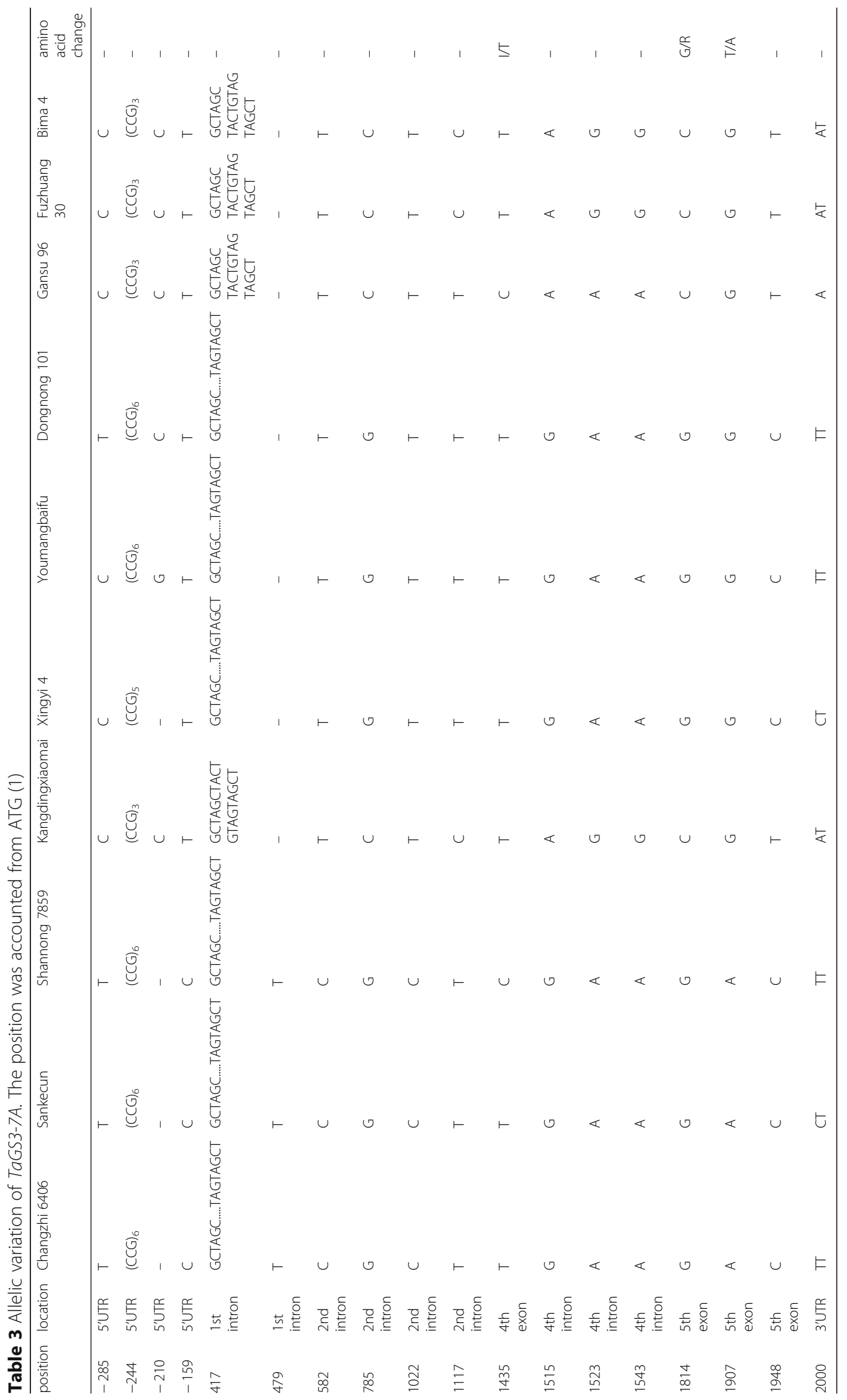




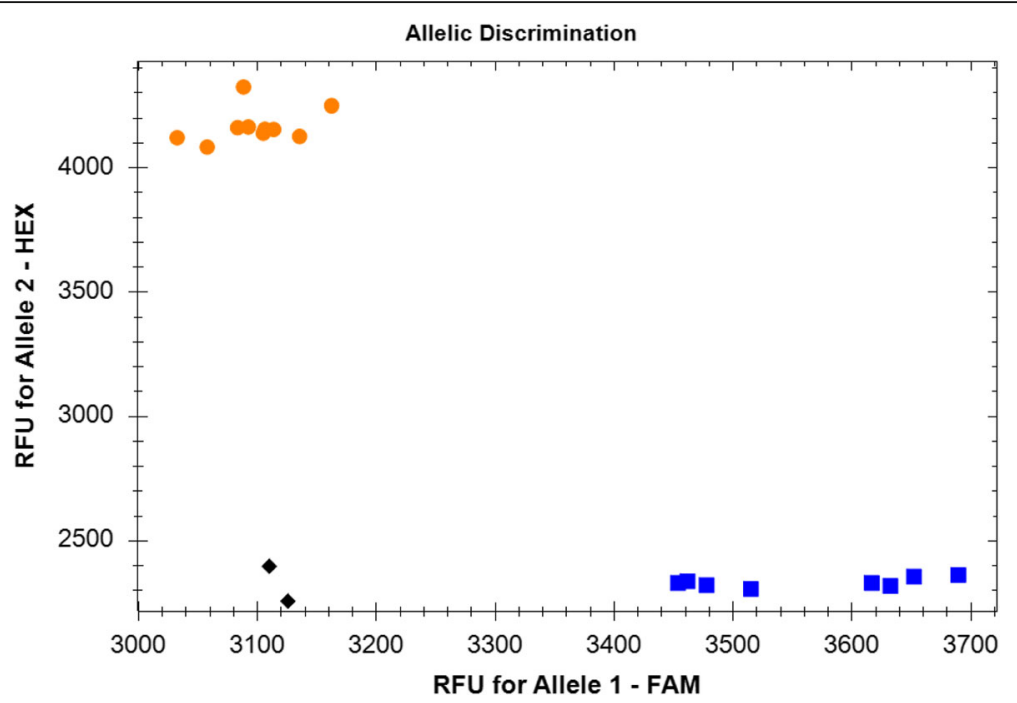

Fig. 3 Scatter plots for KASP assays for TaGS3-7A showing clustering of varieties on the X- (FAM) and Y- (HEX) axes. Varieties colored blue have the FAM-type allele, for which the clustered samples carried TaGS3-7A-G allele showed small grain weight value and grain size; varieties colored orange have the HEX-type allele, the clustered samples carried TaGS3-7A-A allele showed larger grain weight value and grain size; black dots represent the NTC (no template control)

degradation of the GS3 protein in rice [45]. Therefore, evolution of GS3 has led to differences in the length of GS3 in monocotyledons and likely fine-tune of the protein function.

\section{Polymorphism of GS3 and its association with grain weight}

In the present study, the isolated wheat TaGS3-4A, TaGS3-7A, and TaGS3-7D genes were predicted to contain five exons and four introns, which are identical to GS3 genes in rice. A large number of variations for TaGS3-7A and TaGS3-4A were detected. Only one variant located in the GS3 Gy-subunit was detected in TaGS3-4A and this variant seemed to be not associated with grain weight in the different phenotype pools. Nevertheless, the non-conserved domain of GS3 is involved in positive regulation of grain length in rice [46]. Additional polymorphic loci motifs of (AT) $\mathrm{n}$ in the fourth intron and (TCC) $n$ in the fifth exon of GS3 were mainly associated with medium to short grains among Chinese rice accessions because 13 accessions harboring the TGC mutation produced long grains, which suggested that the C-A mutation did not completely explain the accessions with long or short grains in Chinese rice germplasm. In maize, two polymorphisms in the fifth exon (not located within the conserved region) and the promoter region are significantly associated with kernel length and 1000-kernel weight In similarity, we also found that variations in the conserved domain region was not associated with grain weight in consideration of allelic frequency between two pools with different phenotype. However, an additional SNP located in the cysteine-rich region that showed a difference in frequencyand thus applied for marker development. This variation probably effect $G \beta \gamma$ dimers from autoinhibition through alternation of the structure of cysteine-rich region $[45,47]$. The cysteine-rich region plays a variety of roles in exercise its function. GAST1-like proteins are involved in redox reactions via their cysteine-rich domain and GASA4 mutated by replacement of conserved cysteines with alanines lost its redox activity and the capability to promote gibberellin responses [48]. In addition, the C-terminal cysteine-rich region is sufficient for rice OsDep1 to confer cadmium tolerance to yeast cells [49]. Collectively, the afore-mentioned findings suggest that variation in the non-conserved domains of GS3 may also affect the phenotype.

In previous studies, one QTL for grain weight was indicated to be associated with TaGS3-7D in wheat and all variation between parental lines was contained in the second intron. However, subgenomes A and B show broader genetic diversity compared with that of $\mathrm{D}$ subgenome in hexaploid wheat, leading to assessment of the sequence diversity of TaGS3 and development of a functional marker for TaGS3 for use in breeding $[39,50]$. However, we observed that none of the reported major QTLs for grain weight/size were located in the terminal region of chromosome arm 7AS in the vicinity of TaGS3-7A, which was probably because cultivars often carry a weak allelic variation of functional genes, which could provide balance for maintaining the enhancement of grain weight and plant fitness $[45,51,52]$. However, variations with weak effects on phenotype was usually behind high threshold in QTL mapping. Therefore, 
Table 4 Association analysis of kernel traits between TaGS3-7A$G$ and TaGS3-7A-A genotypes. note: ${ }^{*} p<0.05,{ }^{* *} p<0.01$

\begin{tabular}{|c|c|c|c|c|}
\hline Trait & Allele & $\mathrm{N}$ & Mean & Sig \\
\hline \multirow[t]{2}{*}{ TKW_E1 } & TaGS3-7A-G & 116 & $36.40 \pm 0.69$ & $0.010^{* *}$ \\
\hline & TaGS3-7A-A & 20 & $40.96 \pm 1.28$ & \\
\hline \multirow[t]{2}{*}{ TKW_E2 } & TaGS3-7A-G & 140 & $32.76 \pm 0.61$ & 0.075 \\
\hline & TaGS3-7A-A & 21 & $35.77 \pm 1.44$ & \\
\hline \multirow[t]{2}{*}{ TKW_E3 } & TaGS3-7A-G & 145 & $34.58 \pm 0.61$ & $0.004^{* *}$ \\
\hline & TaGS3-7A-A & 24 & $39.11 \pm 1.22$ & \\
\hline \multirow[t]{2}{*}{ GL_E1 } & TaGS3-7A-G & 147 & $0.639 \pm 0.005$ & 0.27 \\
\hline & TaGS3-7A-A & 24 & $0.654 \pm 0.013$ & \\
\hline \multirow[t]{2}{*}{ GL_E2 } & TaGS3-7A-G & 143 & $0.643 \pm 0.005$ & 0.089 \\
\hline & TaGS3-7A-A & 22 & $0.665 \pm 0.011$ & \\
\hline \multirow[t]{2}{*}{ GL_E3 } & TaGS3-7A-G & 145 & $0.668 \pm 0.005$ & 0.134 \\
\hline & TaGS3-7A-A & 24 & $0.686 \pm 0.010$ & \\
\hline \multirow[t]{2}{*}{ GW_E1 } & TaGS3-7A-G & 147 & $0.303 \pm 0.003$ & $0.013^{*}$ \\
\hline & TaGS3-7A-A & 24 & $0.321 \pm 0.006$ & \\
\hline \multirow[t]{2}{*}{ GW_E2 } & TaGS3-7A-G & 143 & $0.301 \pm 0.002$ & $0.007^{* *}$ \\
\hline & TaGS3-7A-A & 22 & $0.316 \pm 0.005$ & \\
\hline \multirow[t]{2}{*}{ GW_E3 } & TaGS3-7A-G & 145 & $0.306 \pm 0.002$ & $0.020^{*}$ \\
\hline & TaGS3-7A-A & 24 & $0.318 \pm 0.006$ & \\
\hline \multirow[t]{2}{*}{ GT_E1 } & TaGS3-7A-G & 147 & $0.267 \pm 0.002$ & $0.010^{* *}$ \\
\hline & TaGS3-7A-A & 24 & $0.281 \pm 0.004$ & \\
\hline \multirow[t]{2}{*}{ GT_E2 } & TaGS3-7A-G & 143 & $0.278 \pm 0.002$ & $0.005^{* *}$ \\
\hline & TaGS3-7A-A & 22 & $0.292 \pm 0.004$ & \\
\hline \multirow[t]{2}{*}{ GT_E3 } & TaGS3-7A-G & 145 & $0.289 \pm 0.002$ & 0.083 \\
\hline & TaGS3-7A-A & 24 & $0.298 \pm 0.004$ & \\
\hline \multirow[t]{2}{*}{ GL/GW_E1 } & TaGS3-7A-G & 147 & $2.119 \pm 0.019$ & 0.221 \\
\hline & TaGS3-7A-A & 24 & $2.053 \pm 0.059$ & \\
\hline \multirow[t]{2}{*}{ GL/GW_E2 } & TaGS3-7A-G & 143 & $2.146 \pm 0.016$ & 0.456 \\
\hline & TaGS3-7A-A & 22 & $2.113 \pm 0.044$ & \\
\hline \multirow[t]{2}{*}{ GL/GW_E3 } & TaGS3-7A-G & 145 & $2.190 \pm 0.016$ & 0.784 \\
\hline & TaGS3-7A-A & 24 & $2.178 \pm 0.053$ & \\
\hline
\end{tabular}

application of natural accessions with extreme phenotype as a sampling was an alternatively method for detection of functional allelic variation in wheat $[31,53,54]$.

In particular, modern cultivars grown in the main wheat-production areas of China showed a different allele frequency of TaGS3-7A-A and exceeded more than $50 \%$ of cultivars in most provinces (Fig. 4). The highest frequency of TaGS3-7A-A and TaGS5-3A-T alleles were detected in southwestern China, which indicated that the alleles were probably strongly selected in this region [2]. In addition, the lowest frequency of the TaGS3-7A$A$ allele was observed in Henan province, which suggested that there remains potential for improvement of grain weight in some wheat-production areas by utilization of the TaGS3-7A-A allele.
Cloning of grain weight-related genes and characterization of allelic variation in wheat are essential for molecular breeding. In addition, the functional marker for TaGS3-7A developed in the present study is potentially useful for wheat yield improvement.

\section{Conclusions}

In the present study, we cloned homologs of TaGS3 in hexaploid wheat, systematically evaluated the evolutionary progress of GS3 in plant species, and designed a functional marker for TaGS3-7A. Wheat carries three TaGS3 homologs, for which the amino acid sequences contain a similar atypical $\mathrm{G} \gamma$ domain and a cysteine-rich region that differed in length from that of rice. On the basis of sequence comparison between two extreme phenotypic pools, a KASP marker was designed and shown to have significant genetic effects on grain weight, kernel width, and kernel thickness. The present results provide novel molecular information on GS3 and the designed functional marker may be useful in genetic improvement programs for wheat.

\section{Methods \\ Plant materials}

Wheat (Triticum aestivum) 'Changzhi 6406', which is characterized by a high grain weight in previous, was used for isolation of sequences for the three GS3 homologs. Ten wheat accessions, divided into two pools, were used for TaGS3-4A and TaGS3-7A gene cloning; one pool comprised accessions with high grain weights ('Changzhi 6406', 'Sankecun', 'Shannong 7859', 'Kangdingxiaomai', and 'Xingyi 4') and the second pool consisted of accessions with low grain weight ('Youmangbaifu', 'Dongnong 101', 'Gansu 96', 'Fuzhuang 30', and 'Bima 4') [2].

The MCC germplasm collection, comprising 224 wheat accessions representing high genetic diversity in China, were used for validation of the association of the genetic marker and kernel-related traits (kernel length, kernel width, kernel thickness, and 1000-kernel weight) in three independent environments (E1: Luoyang at 2002; E2 Luoyang at 2005; E3 Shunyi at 2010) [2].

A panel of 238 modern wheat cultivars grown in the main wheat-production areas of China and released in the twenty-first century were used to investigate the allelic frequency and distribution of TaGS3-7A-A. These accessions including the 75 accessions in southwest of China and another previously characterized 163 accessions in the central plateau and north of the Yellow River [35].

\section{Gene isolation and analysis}

TaGS3 homologous gene sequences were isolated using a comparative genomics method. GS3 gene sequences 


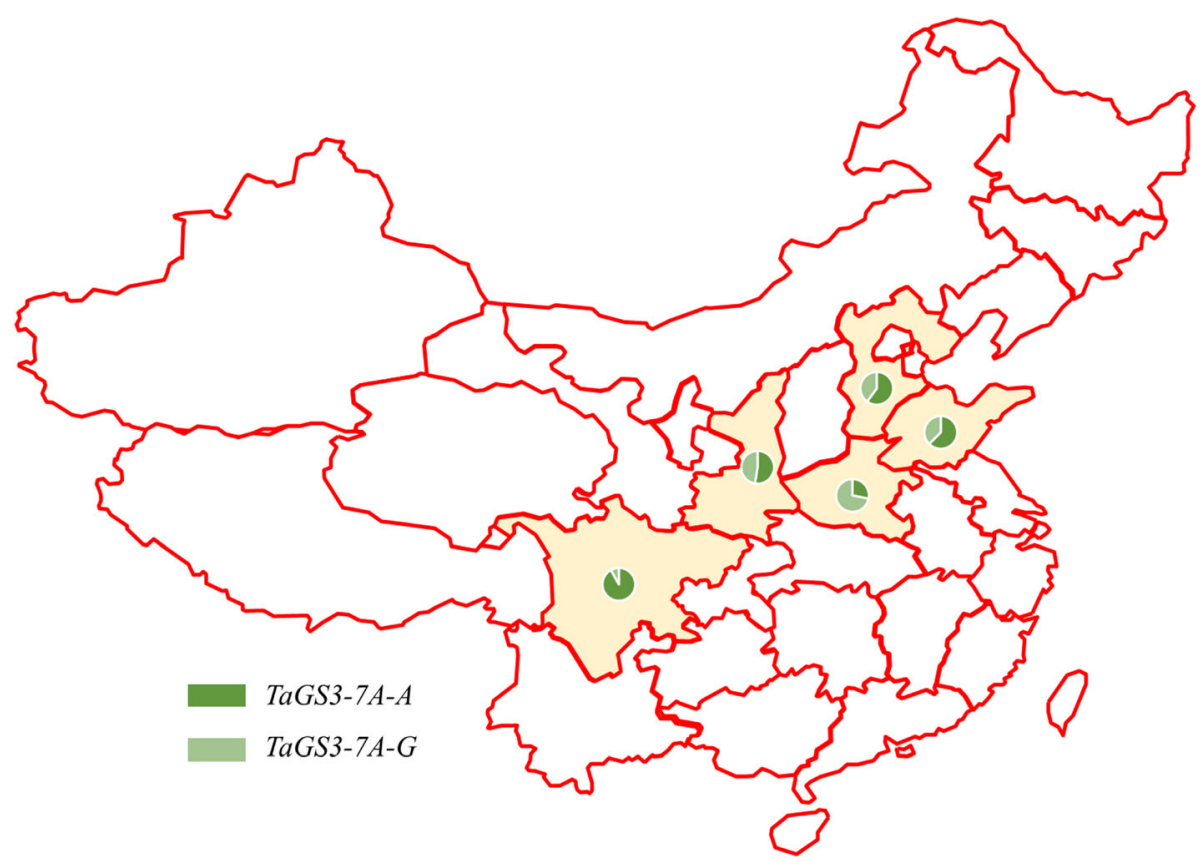

Fig. 4 Distribution of the TaGS3-7A-A and TaGS3-7A-G allele in the main wheat production areas of China

for rice and wheat (GenBank accession numbers DQ355996 and KF687956, respectively) were used to search for orthologs in the wheat genome using the Ensembl Plants online resource (http://plants.ensembl. org/Triticum_aestivum/Tools/Blast?db=core). Primer sets to target a specific region for each sequence were designed with Oligo 7 software and synthesized by Tsingke Biological Technology Co., Ltd. (Beijing, China).

Since TaGS3 gene containing high GC content and repetitive sequence, Taq DNA polymerase with GC Buffer (probably lose some fidelity. Takara, RR02AG) were selected for PCR reaction. Amplification of the gene sequences by PCR was performed in a volume of $30 \mu \mathrm{l}$, which contained $15 \mu \mathrm{l}$ of $2 \times \mathrm{GC}$ buffer I, $10 \mu \mathrm{M}$ dNTPs, $20 \mu \mathrm{mol}$ of each primer, $1 \mathrm{U}$ LA Taq ${ }^{\mathrm{mm}}$ and $\sim 100 \mathrm{ng}$ template DNA. All reagents were obtained from Takara Biotechnology Co., Ltd. (http:// www.takara.com.cn). The reaction protocol was $94{ }^{\circ} \mathrm{C}$ for $5 \mathrm{~min}$, followed by $35 \mathrm{cycles}$ of $94^{\circ} \mathrm{C}$ for $45 \mathrm{~s}$, $58^{\circ} \mathrm{C}$ for $45 \mathrm{~s}$, and $72{ }^{\circ} \mathrm{C}$ for $2 \mathrm{~min} 30 \mathrm{~s}$, and final extension at $72{ }^{\circ} \mathrm{C}$ for $10 \mathrm{~min}$.

The PCR products were separated by electrophoresis on $2 \%$ agarose gels. All bands were cleaned and cloned into the pEASY-T5 Zero vector (Beijing TransGen Biotech Co., Ltd.; http://www.transgen.com. cn) and transformed into DH5 $\alpha$ competent Escherichia coli cells using the heat shock method. Single clones were sequenced by Tsingke Biotech Co., Ltd. Sequence alignment and assembly was performed using DNAMAN software (http://www.lynnon.com).
All sequenced clones were aligned against the wheat reference genome (IWGSC v1.0) to confirm their chromosomal locations.

\section{Phylogenetic analysis}

The rice GS3 (Os03g0407400) ortholog sequence was used to search genomic databases for common higher plants (e.g., rice, maize, Arabidopsis, soybean, sorghum, and medicago) in Ensembl Plants website (http://plants. ensembl.org/Triticum_aestivum/Tools/Blast?db=core).

The gene OsDep1, which encodes a G protein, was used as the paralogous outgroup. Alignment of the deduced amino acids sequences was performed using MUSCLE in MEGA 7.0 software [55]. A phylogenetic tree was constructed using the neighbor-joining method, Poisson model, uniform rate, and partial deletion (site coverage off 50\%) as implemented in MEGA [56]. A bootstrap analysis was performed to test the robustness of the phylogenetic construction with 1000 replications.

\section{SNP mining and development of a gene-specific KASP marker}

Single-nucleotide polymorphisms that caused a putative amino acid change and differed in frequency between the grain weight accessions were used for marker development.

For the KASP genotyping procedure, the $10 \mu \mathrm{l}$ PCR mixture contained $100 \mathrm{ng}$ template DNA, $5 \mu \mathrm{l}$ KASP Master mix, $4 \mathrm{mM} \mathrm{MgCl}_{2}$, and $1.4 \mu \mathrm{l}$ primer mixture,

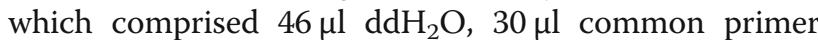
$(10 \mu \mathrm{M})$, and $12 \mu \mathrm{l}$ of each tailed primer $(10 \mu \mathrm{M})$. The 
PCR cycling protocol consisted of a hot start at $95^{\circ} \mathrm{C}$ for $15 \mathrm{~min}$, followed by ten touchdown cycles $\left(95^{\circ} \mathrm{C}\right.$ for 20 $\mathrm{s}$; touchdown at $65^{\circ} \mathrm{C}$ initially and decreasing by $-1{ }^{\circ} \mathrm{C}$ per cycle for $60 \mathrm{~s}$ ), followed by 30 additional cycles of annealing $\left(95^{\circ} \mathrm{C}\right.$ for $10 \mathrm{~s} ; 57^{\circ} \mathrm{C}$ for $\left.60 \mathrm{~s}\right)$. The extension step (each three cycles of annealing: $95^{\circ} \mathrm{C}$ for $10 \mathrm{~s} ; 57^{\circ} \mathrm{C}$ for $60 \mathrm{~s}$ ) was repeated three times to obtain the best genotype clustering results $[35,57]$. The last $37^{\circ} \mathrm{C}$ for 1 min was set up for collection of HEX and FAM fluorescence signal value. Final signal detection was applied under Bio-Rad CFX Connect ${ }^{\text {ti }}$ PCR system and allele discrimination plots (clustering) generated with Bio-Rad CFX Manager ${ }^{\text {rus }}$ software. The primers were synthesized by Tsingke Biological Technology Co., Ltd., and the developed KASP marker was used to screen all 224 MCC germplasm and 238 modern wheat cultivars in China.

\section{Statistical analysis}

One-way analysis of variance was performed using IBM SPSS Statistics for Windows version 22.0 (IBM Corporation, Armonk, NY, USA) to determine the significance of differences in grain traits between the two alleles. Phenotypic data recorded in three independent environments (E1: Luoyang at 2002; E2: Luoyang at 2005; E3: Beijing at 2010) were derived from a previous study [26]. Accessions either with indistinct genotyping signal or lacking phenotype data were excluded from analyses.

\section{Supplementary information}

Supplementary information accompanies this paper at https://doi.org/10. 1186/s12863-019-0800-6.

Additional file 1: Table S1. Allelic variation of TaGS3-7A in Chinese mini-core collection based on Kompetitive allele-specific PCR (KASP) marker designed for the SNP at position 1907 (A/G). Table S2. Allelic variation of TaGS3-7A in main wheat production areas (Hebei, Shandong, Shaanxi, Henan and Sichuan) in China based on Kompetitive allelespecific PCR (KASP) marker designed for the SNP at position 1907 (A/G).

\section{Abbreviations}

ALA: Alanine; bp: Base pair; GS3: Grain shape 3; KASP: Kompetitive AlleleSpecific PCR; MEGA: Molecular Evolutionary Genetic Analysis; PCR: Polymerase Chain Reaction; QTL: Quantitative trait locus; SNP: Single Nucleotide Polymorphism; THR: Threonine; UTR: Untranslated region

\section{Acknowledgments}

We gratefully acknowledge Prof. Xueyong Zhang for kindly providing the mini-core germplasm collection.

\section{Authors' contributions}

ZL, TC, JY and QW conceived the study. JY designed the experiments. JY, YZ, WH and $Y C$ performed gene cloning, SNP mining and KASP marker design. $Y Z, H Z$ and XW conducted the phylogenetic analysis. GG performed the allele frequency analysis. JY and ZL wrote the manuscript. All authors read and approved the manuscript.

\section{Funding}

This research was supported by the Special Fund for Henan Agricultural Research System (S2010-01-G03) and this funding played no role in the design of the study and collection, analysis, and interpretation of data and in writing the manuscript.

\section{Availability of data and materials}

All data generated or analyzed during this study are included in this published article (and additional files). The kernel traits of mini-core collection (MCC) germplasms are derived from the following public publication: Ma L, Li T, Hao C, Wang Y, Chen X, Zhang X: TaGS5-3A, a grain size gene selected during wheat improvement for larger kernel and yield. Plant Biotechnol J 2016, 14(5):1269-1280. https://doi.org/10.1111/pbi.12492.

Ethics approval and consent to participate

Not applicable.

\section{Consent for publication}

Not applicable.

\section{Competing interests}

The authors declare that the research was conducted in the absence of any commercial or financial relationships that could be construed as a potential conflict of interest.

Received: 18 June 2019 Accepted: 12 December 2019 Published online: 18 December 2019

\section{References}

1. Lopes MS, Reynolds MP, Manes Y, Singh RP, Crossa J, Braun HJ. Genetic yield gains and changes in associated traits of CIMMYT spring bread wheat in a "historic" set representing 30 years of breeding. Crop Sci. 2012;52(3): 1123-31.

2. Ma L, Li T, Hao C, Wang Y, Chen X, Zhang X. TaGS5-3A, a grain size gene selected during wheat improvement for larger kernel and yield. Plant Biotechnol J. 2016:14(5):1269-80.

3. Slafer GA. Genetic basis of yield as viewed from a crop physiologist's perspective. Ann Appl Biol. 2003;142(2):117-128.

4. Zuo J, Li J. Molecular genetic dissection of quantitative trait loci regulating rice grain size. Annu Rev Genet. 2014:48:99-118.

5. Li N, Xu R, Li Y. Molecular networks of seed size control in plants. Annu Rev Plant Biol. 2019:70:435-63.

6. Fan C, Xing Y, Mao H, Lu T, Han B, Xu C, Li X, Zhang Q. GS3, a major QTL for grain length and weight and minor QTL for grain width and thickness in rice, encodes a putative transmembrane protein. Theor Appl Genet. 2006; 112(6):1164-71.

7. Fan C, Yu S, Wang C, Xing Y. A causal C-A mutation in the second exon of GS3 highly associated with rice grain length and validated as a functional marker. Theor Appl Genet. 2009:118(3):465-472.

8. Xia D, Zhou H, Liu R, Dan W, Li P, Wu B, Chen J, Wang L, Gao G, Zhang Q, et al. GL3.3, a novel QTL encoding a GSK3/SHAGGY-like kinase, Epistatically interacts with GS3 to produce extra-long grains in Rice. Mol Plant. 2018; 11(5):754-56.

9. Li Y, Fan C, Xing Y, Jiang Y, Luo L, Sun L, Shao D, Xu C, Li X, Xiao J, et al. Natural variation in GS5 plays an important role in regulating grain size and yield in rice. Nat Genet. 2011;43(12):1266-69.

10. Takano-Kai N, Jiang H, Kubo T, Sweeney M, Matsumoto T, Kanamori H, Padhukasahasram B, Bustamante C, Yoshimura A, Doi K, et al. Evolutionary history of GS3, a gene conferring grain length in rice. Genet. 2009;182(4): 1323-34.

11. Weng J, Gu S, Wan X, Gao H, Guo T, Su N, Lei C, Zhang X, Cheng Z, Guo X, et al. Isolation and initial characterization of GW5, a major QTL associated with rice grain width and weight. Cell Res. 2008;18(12):1199-1209.

12. Liu J, Chen J, Zheng X, Wu F, Lin Q, Heng Y, Tian P, Cheng Z, Yu X, Zhou K, et al. GW5 acts in the brassinosteroid signalling pathway to regulate grain width and weight in rice. Nat Plants. 2017:3:17043.

13. Si L, Chen J, Huang X, Gong H, Luo J, Hou Q, Zhou T, Lu T, Zhu J, Shangguan $Y$, et al. OsSPL13 controls grain size in cultivated rice. Nat Genet. 2016:48:447.

14. Song XJ, Kuroha T, Ayano M, Furuta T, Nagai K, Komeda N, Segami S, Miura K, Ogawa D, Kamura T, et al. Rare allele of a previously unidentified histone H4 acetyltransferase enhances grain weight, yield, and plant biomass in rice. Proc Natl Acad Sci. 2015;112(1):76-81.

15. Song XJ, Huang W, Shi M, Zhu MZ, Lin HX. A QTL for rice grain width and weight encodes a previously unknown RING-type E3 ubiquitin ligase. Nat Genet. 2007;39(5):623-30. 
16. Xia T, Li N, Dumenil J, Li J, Kamenski A, Bevan MW, Gao F, Li Y. The ubiquitin receptor DA1 interacts with the E3 ubiquitin ligase DA2 to regulate seed and organ size in Arabidopsis. Plant Cell. 2013;25(9):3347-59.

17. Xia D, Zhou H, Liu R, Dan W, Li P, Wu B, Chen J, Wang L, Gao G, Zhang Q, et al. GL3.3, a Novel QTL Encoding a GSK3/SHAGGY-like Kinase, Epistatically Interacts with GS3 to Form Extra-long Grains in Rice. Mol Plant. 2018;11(5): 754-56.

18. Qi P, Lin YS, Song XJ, Shen JB, Huang W, Shan JX, Zhu MZ, Jiang L, Gao JP, Lin HX. The novel quantitative trait locus GL3.1 controls rice grain size and yield by regulating Cyclin-T1;3. Cell Res. 2012;22(12):1666-80.

19. Gao X, Zhang J-Q, Zhang X, Zhou J, Jiang Z, Huang P, Tang Z, Bao Y, Cheng J, Tang $H$, et al. Rice qGL3/OsPPKL1 functions with the GSK3/SHAGGY-like kinase OsGSK3 to modulate Brassinosteroid signaling. Plant Cell. 2019;31(5): 1077-93.

20. Zhang X, Wang J, Huang J, Lan H, Wang C, Yin C, Wu Y, Tang H, Qian Q, L $J$, et al. Rare allele of OsPPKL1 associated with grain length causes extralarge grain and a significant yield increase in rice. Proc Natl Acad Sci U S A. 2012;109(52):21534-539.

21. Huang X, Qian Q, Liu Z, Sun H, He S, Luo D, Xia G, Chu C, Li J, Fu X. Natural variation at the DEP1 locus enhances grain yield in rice. Nat Genet. 2009; 41(4):494-97.

22. Appels REK, Feuillet C. Shifting the limits in wheat research and breeding using a fully annotated reference genome. Sci. 2018;361(6403):eaar7191.

23. Brenchley R, Spannagl M, Pfeifer M, Barker GLA, D'Amore R, Allen AM, McKenzie N, Kramer M, Kerhornou A, Bolser D, et al. Analysis of the bread wheat genome using whole-genome shotgun sequencing. Nat. 2012;491:705.

24. Kurata N, Moore G, Nagamura Y, Foote T, Yano M, Minobe Y, Gale M. Conservation of genome structure between Rice and wheat. Nat Biotechnol. 1994;12(3):276-78.

25. Su Z, Hao C, Wang L, Dong Y, Zhang X. Identification and development of a functional marker of TaGW2 associated with grain weight in bread wheat (Triticum aestivum L.). Theor Appl Genet. 2011;122(1):211-23.

26. Qin L, Hao C, Hou J, Wang Y, Li T, Wang L, Ma Z, Zhang X. Homologous haplotypes, expression, genetic effects and geographic distribution of the wheat yield gene TaGW2. BMC Plant Biol. 2014;14(1):107.

27. Zhang Y, Li D, Zhang D, Zhao X, Cao X, Dong L, Liu J, Chen K, Zhang H, Gao C, et al. Analysis of the functions of TaGW2 homoeologs in wheat grain weight and protein content traits. Plant J. 2018;94(5):857-66.

28. Sestili F, Pagliarello R, Zega A, Saletti R, Pucci A, Botticella E, Masci S, Tundo S, Moscetti I, Foti S, et al. Enhancing grain size in durum wheat using RNAi to knockdown GW2 genes. Theor Appl Genet. 2019;132(2):419-29.

29. Hong Y, Chen L, Du LP, Su Z, Wang J, Ye X, Qi L, Zhang Z. Transcript suppression of TaGW2 increased grain width and weight in bread wheat. Funct Integr Genomics. 2014;14(2):341-49.

30. Wang S, Zhang X, Chen F, Cui D. A single-nucleotide polymorphism of TaGS5 gene revealed its association with kernel weight in Chinese bread wheat. Front Plant Sci. 2015;6:1166

31. Hanif M, Gao F, Liu J, Wen W, Zhang Y, Rasheed A, Xia X, He Z, Cao S. TaTGW6-A1, an ortholog of rice TGW6, is associated with grain weight and yield in bread wheat. Mol Breed. 2015;36(1):1.

32. Hu M-J, Zhang H-P, Cao J-J, Zhu X-F, Wang S-X, Jiang H, Wu ZY, Lu J, Chang $C$, Sun $G-L$, et al. Characterization of an IAA-glucose hydrolase gene TaTGW6 associated with grain weight in common wheat (Triticum aestivum L.). Mol Breeding. 2016;36(3):25

33. Wang W, Pan Q, Tian B, He F, Chen Y, Bai G, Akhunova A, Trick HN, Akhunov E. Gene editing of the wheat homologs of TONNEAU1-recruiting motif encoding gene affects grain shape and weight in wheat. Plant J. 2019.

34. Ma J, Ding P, Qin P, Liu Y-X, Xie Q, Chen G, Li W, Jiang Q, Chen G, Lan X-J, et al. Structure and expression of the TaGW7 in bread wheat (Triticum aestivum L.). Plant Growth Regul. 2017;82(2):281-91.

35. Yang J, Zhou Y, Wu Q, Chen Y, Zhang P, Zhang Y, Hu W, Wang X, Zhao H, Dong $L$, et al. Molecular characterization of a novel TaGL3-5A allele and its association with grain length in wheat (Triticum aestivum L.). Theor Appl Genet. 2019;132(6):1799-1814.

36. Zhai H, Feng Z, Du X, Song Y, Liu X, Qi Z, Song L, Li J, Li L, Peng H, et al. A novel allele of TaGW2-A1 is located in a finely mapped QTL that increases grain weight but decreases grain number in wheat (Triticum aestivum L.). Theor Appl Genet. 2018;131(3):539-53.

37. Yan X, Zhao L, Ren Y, Dong Z, Cui D, Chen F. Genome-wide association study revealed that the TaGW8 gene was associated with kernel size in Chinese bread wheat. Sci Rep. 2019;9(1):2702.
38. Zhang Y, Liu J, Xia X, He Z. TaGS-D1, an ortholog of rice OsGS3, is associated with grain weight and grain length in common wheat. Mol Breed. 2014;34(3):1097-1107.

39. Akhunov ED, Akhunova AR, Anderson OD, Anderson JA, Blake N, Clegg MT, Coleman-Derr D, Conley EJ, Crossman CC, Deal KR, et al. Nucleotide diversity maps reveal variation in diversity among wheat genomes and chromosomes. BMC Genomics. 2010;11(1):702.

40. Wang S, Wong D, Forrest K, Allen A, Chao S, Huang BE, Maccaferri M, Salvi S, Milner SG, Cattivelli L, et al. Characterization of polyploid wheat genomic diversity using a high-density 90,000 single nucleotide polymorphism array. Plant Biotechnol J. 2014;12(6):787-96.

41. Devos KM, Dubcovsky J, Dvorák J, Chinoy CN, Gale MD. Structural evolution of wheat chromosomes $4 \mathrm{~A}, 5 \mathrm{~A}$, and $7 \mathrm{~B}$ and its impact on recombination. Theor Appl Genet. 1995;91(2):282-88.

42. Berkman PJ, Skarshewski A, Manoli S, Lorenc MT, Stiller J, Smits L, Lai K, Campbell E, Kubalakova M, Simkova $H$, et al. Sequencing wheat chromosome arm 7BS delimits the 7BS/4AL translocation and reveals homoeologous gene conservation. Theor Appl Genet. 2012;124(3):423-32.

43. Hernandez P, Martis M, Dorado G, Pfeifer M, Galvez S, Schaaf S, Jouve N, Simkova H, Valarik M, Dolezel J, et al. Next-generation sequencing and syntenic integration of flow-sorted arms of wheat chromosome 4A exposes the chromosome structure and gene content. Plant J. 2012;69(3):377-86.

44. Akpinar BA, Biyiklioglu S, Alptekin B, Havrankova M, Vrana J, Dolezel J, Distelfeld A, Hernandez P, Iwgsc, Budak H. Chromosome-based survey sequencing reveals the genome organization of wild wheat progenitor Triticum dicoccoides. Plant Biotechnol J. 2018;16(12):2077-87.

45. Sun S, Wang L, Mao H, Shao L, Li X, Xiao J, Ouyang Y, Zhang Q. A G-protein pathway determines grain size in rice. Nat Commun. 2018;9(1):851.

46. Mao H, Sun S, Yao J, Wang C, Yu S, Xu C, Li X, Zhang Q. Linking differential domain functions of the GS3 protein to natural variation of grain size in rice. Proc Natl Acad Sci U S A. 2010;107(45):19579-584

47. Urano D, Miura K, Wu Q, Iwasaki Y, Jackson D, Jones AM. Plant morphology of Heterotrimeric G protein mutants. Plant Cell Physiol. 2016;57(3):437-45.

48. Rubinovich $L$, Weiss $D$. The Arabidopsis cysteine-rich protein GASA4 promotes GA responses and exhibits redox activity in bacteria and in planta. Plant J. 2010;64(6):1018-27.

49. Kunihiro S, Saito T, Matsuda T, Inoue M, Kuramata M, Taguchi-Shiobara F, Youssefian S, Berberich T, Kusano T. Rice DEP1, encoding a highly cysteinerich $\mathrm{G}$ protein gamma subunit, confers cadmium tolerance on yeast cells and plants. J Exp Bot. 2013;64(14):4517-27.

50. Gutierrez-Gonzalez JJ, Mascher M, Poland J, Muehlbauer GJ. Dense genotyping-by-sequencing linkage maps of two synthetic W7984×Opata reference populations provide insights into wheat structural diversity. Sci Rep. 2019;9(1):1793.

51. Li Y, Xu M. CCT family genes in cereal crops: a current overview. Crop J. 2017:5(6):449-58.

52. Sakuma S, Golan G, Guo Z, Ogawa T, Tagiri A, Sugimoto K, Bernhardt N, Brassac J, Mascher M, Hensel G, et al. Unleashing floret fertility in wheat through the mutation of a homeobox gene. Proc Natl Acad Sci U S A. 2019; 116(11):5182-87.

53. Sajjad M, Ma X, Habibullah Khan S, Shoaib M, Song Y, Yang W, Zhang A, Liu D. TaFlo2-A1, an ortholog of rice Flo2, is associated with thousand grain weight in bread wheat (Triticum aestivum L.). BMC Plant Biol. 2017;17(1):164.

54. Zhang $Y$, Xia X, He Z. The seed dormancy allele TaSdr-A1a associated with pre-harvest sprouting tolerance is mainly present in Chinese wheat landraces. Theor Appl Genet. 2017;130(1):81-89.

55. Edgar RC. MUSCLE: multiple sequence alignment with high accuracy and high throughput. Nucleic Acids Res. 2004;32(5):1792-97.

56. Kumar S, Stecher G, Tamura K. MEGA7: molecular evolutionary genetics analysis version 7.0 for bigger datasets. Mol Biol Evol. 2016;33(7):1870-74.

57. Rasheed A, Wen W, Gao F, Zhai S, Jin H, Liu J, Guo Q, Zhang Y, Dreisigacker $S$, Xia X, et al. Development and validation of KASP assays for genes underpinning key economic traits in bread wheat. Theor Appl Genet. 2016; 129(10):1843-60.

\section{Publisher's Note}

Springer Nature remains neutral with regard to jurisdictional claims in published maps and institutional affiliations. 